COMMENTARY

\title{
When is HRT the Solution?
}

\author{
Nancy L. Belcher, PhD., MPA*
}

Department of Womens Health, North American Menopause Society, United States of America

*Corresponding author: Nancy L. Belcher, PhD., MPA, Department of Womens Health, North American Menopause Society, United States of America

Menopause, and the phase that leads up to it, called perimenopause, are normal and natural events that can start anywhere between 35-55 years of age. Menopause and perimenopause are transition periods wherein the levels of estrogen, progesterone, and testosterone decrease. Replacing those lost hormones is easy with hormone replacement therapy (HRT). HRT in menopause has been proven the most effective treatment for many menopause symptoms.

While there is no 'treatment' for menopause, there are ways to treat the often-chronic symptoms that result from menopause. Menopause is a positive beginning, with opportunities to take preventive action against major health risks associated with decreased hormones as we age. HRT in menopause can allow you the opportunity to replace the changing levels of estrogen, progesterone, and testosterone and finally provide the most effective way to regulate the highs and lows of hormones that you've endured most of your life.

Sadly, not enough women are talking about the symptoms of peri- and menopause, and there is a lack of credible scientific information about solutions to their often life-altering menopause symptoms. Winona hopes to change that by sharing the latest science information available to treat symptoms, and preventing long-term health problems. There is no benefit to simply 'enduring' menopause.

Yes, we've read the jokes about hot flashes and moodiness with 'the change,' but peri- and menopause can cause much more serious problems like weight gain, depression, decreased libido, and fatigue - all of which can profoundly impact your quality of life.

As we age, our hormones change and hormone replacement therapy (HRT) might be the way to help you relieve many of your menopause symptoms. Each woman will require her own specially blended 'hormone cocktail' to help relieve her symptoms. Just like how we each experienced puberty differently, we will experience menopause differently too.

\section{What is HRT?}

Winona offers hormone replacement therapy (HRT) to help provide you with the estrogen, progesterone, and testosterone that your body makes less of as you age. You can certainly benefit from natural HRT on your menopause journey.

\section{Estrogen}

Estrogen naturally decreases during menopause, but in an irregular fashion. Estrogen works to "lubricate the body and brain". Taking HRT earlier may improve memory, concentration, and cognition. By adding back the decreasing levels of estrogen, in combination with progesterone and testosterone therapy, you can help ease the symptoms of peri- and menopause and reduce the risk of other diseases such as cancer, osteoporosis, cardiovascular disease, type 2 diabetes, and many more.

Natural estrogen patches, creams, and tablets can help relieve unnecessary menopause symptoms and provide remedies for vaginal dryness, anxiety, and serve as a hot flash treatment. Rather than suffer unnecessarily with the symptoms of menopause, these natural treatments can relieve the symptoms safely and effectively.

Winona provides estrogen that is chemically identical to the kind made by women's ovaries, and described as "bioidentical." Shipped by Winona's private pharmacies

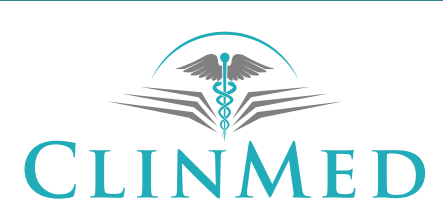

INTERNATIONAL LIBRARY
Citation: Belcher NL (2021) When is HRT the Solution?. Int J Womens Health Wellness 7:125. doi.org/10.23937/2474-1353/1510125

Accepted: September 25, 2021: Published: September 27, 2021

Copyright: (c) 2021 Belcher NL. This is an open-access article distributed under the terms of the Creative Commons Attribution License, which permits unrestricted use, distribution, and reproduction in any medium, provided the original author and source are credited. 
it comes as an FDA-approved pill, patch, or compounded transdermal cream made just for you.

\section{Progesterone}

Once a woman hits her mid to late ' 30 s, she can enter perimenopause and progesterone production in the ovaries starts to decline. Once she reaches menopause, circulating progesterone levels are so low they are similar to levels normally seen in men. Research shows that progesterone supplementation can improve brain function as well as breast, cardiovascular, and nervous system health and decrease the risk of uterine cancer $[1,2]$.

Progesterone has a lot of critical roles including:

- Eases Anxiety, Stress: Has a calming effect on both the mind and the body.

- Improves Mood: Depleted progesterone can cause mood swings and depression.

- Promotes Memory: Progesterone stimulates brain processes and the nervous system.

- Enhances Sleep: Take before bed to help resume normal sleep cycles; it has a sedative effect.

- Helps Regulate Estrogen: If progesterone levels are low, this can throw off estrogen too.

- Helps Prevent Diabetes: Progesterone and estrogen work together to balance the release of insulin.

- Protects from Cancer: Progesterone can help protect against breast and uterine cancers.

- Reduces Weight Gain: Helps the metabolism by contributing to the use of fat for energy.

- Prevents Heart Disease: Assists in preventing plaque from forming on arterial walls.

- Impacts Bone Formation: Essential for bone growth and loss of Calcium.

- Helps Thyroid Gland: Assists in the proper function of the thyroid gland.

- Anti-Aging: Can help to slow down the aging process.

- Improves libido/ sex drive.

- Reduces migraines.

- Reduces inflammation and joint pain.

\section{Testosterone}

Although many think of it as a 'male' hormone, testosterone is also an important female hormone that plays a critical role in women's health [3]. Testosterone maintains normal metabolic function, muscle and bone strength, urogenital health, mood, and cognitive function [3].
A deficiency in testosterone can lead to low sexual desire, arousal, and orgasm. Tiredness, depression, headaches, cognitive problems, osteoporosis, and lean body tissue loss are additional complications from low testosterone [3]. Testosterone levels peak in a woman's '20s and start to drop years before perimenopause. By menopause, most women's levels are half of the peak levels [1]. Winona offers DHEA, a testosterone precursor, to gently yet effectively replace the testosterone that can be missing in the menopausal woman.

\section{Conclusion}

HRT in menopause is the most studied and effective form of treatment to date for hot flashes and night sweats. Scientists have yet to unearth a more effective remedy for menopause symptoms than estrogen, progesterone, and testosterone to improve the profound mind-body shifts that occur during menopause [3-5].

More often than not, women (and sometimes their healthcare providers) don't realize that the cause of their symptoms is the onset of menopause due to declining levels of estrogen, progesterone, and testosterone.

Hormone Replacement Therapy (HRT) can normalize hormone levels by individualizing treatment options. Leading experts recommend HRT at low dose levels in perimenopause and menopause not only to help with symptoms but to prevent long-term disease [4]. Let the Winona women's health care experts work with you to replace the missing ingredients to help you feel, and stay young and healthy.

\section{References}

1. https://www.menopause.org/for-women/sexual-health-menopause-online/changes-at-midlife/changes-in-hormone-levels.

2. https://thebms.org.uk/publications/tools-for-clinicians/testosterone-replacement-in-menopause/.

3. https://www.everlywell.com/blog/testosterone/how-testosterone-levels-impact-your-metabolism-and-your-health/.

4. https://www.oprah.com/health/menopause-research-theoprah-magazine-report-on-hormones/all\#ixzz6eC1UuQOU.

5. https://womeninbalance.org/resources-research/progesterone-and-the-nervous-systembrain/ 